\title{
BACTERIAL ISOLATES ASSOCIATED WITH PELVIC INFLAMMATORY DISEASE AMONG FEMALE PATIENTS ATTENDING SOME HOSPITALS IN ABUJA, NIGERIA.
}

\author{
${ }^{1 *}$ Spencer, T. H. I., ${ }^{2}$ Umeh, P. O., ${ }^{3}$ Irokanulo, E., ${ }^{4}$ Baba, M. M., ${ }^{5}$ Spencer, B. B., ${ }^{1}$ Umar, A. I., ${ }^{3}$ Ardzard, S. A., ${ }^{4}$ Oderinde, \\ S., ${ }^{3}$ Onoja, 0.
}

\author{
${ }^{1}$ Department of Medical Microbiology, Usmanu Danfodiyo University, P.M.B. 2346, Sokoto, Nigeria. \\ ${ }^{2}$ Federal College of Veterinary and Medical Laboratory Technology, Vom, Nigeria. ${ }^{3}$ National Veterinary Research \\ Institute, Vom, Nigeria. ${ }^{4}$ Department of Medical Laboratory Sciences, University of Maiduguri, Nigeria. \\ ${ }^{5}$ Zoology Department, University of Jos, Jos, Nigeria. \\ *E-mail: thispence2005@yahoo.com
}

\begin{abstract}
Background: Pelvic inflammatory disease refers to any infection in the female lower reproductive tract that spreads to the upper reproductive tract. The disease comprises a spectrum of inflammatory disorders of the upper female genital tract, including any combination of endometritis, salpingitis, tubo-ovarian abscess and pelvic peritonitis. PID is not a notifiable disease in most countries, so accurate statistics are not available. This situation is not in any way different here in Nigeria and more so in the Federal Capital Territory, Abuja where this research was conducted, there had never been any published report so far on PID. It therefore became pertinent that such studies be carried out to evaluate the bacterial organisms which may be associated with the disease in this part of Nigeria so that health care providers could take a better look at this affliction in women.

Materials and Methods: Endocervical swabs totalling 100 were aseptically collected from patients with confirmed Pelvic Inflammatory Disease (PID), attending some hospitals in Abuja, Nigeria for detection of bacterial pathogens based on cultural and biochemical characterisation tests. Antibiogram was also conducted on the identified bacterial isolates.

Results: Out of the 100 samples analysed, $43 \%$ yielded pure cultures of bacterial isolates, $2 \%$ yielded mixed cultures while no bacterial growths were recorded from the remaining $55 \%$ samples. Organisms encountered were Staphylococcus aureus (16\%), Escherichia coli (10\%), Streptococcus faecalis (8\%), Pseudomonas aeruginosa (4\%), Streptococcus pyogenes (3\%), Klebsiella pneumoniae (3\%), Proteus rettgeri (2\%) and Proteus mirabilis (1\%). The highest percentage occurrence of pathogenic isolates was observed in polygamous married patients (90\%). The age group most affected falls within the mean age 30.5 years (68\%) while the least affected group falls within the mean age 40.5 years $(5 \%)$. There was a significant difference in the acquisition of PID in relation to marital status $(P<0.05)$. However there was no significant difference in the acquisition of the disease with respect to age $(P>0.05)$. Antibiogram patterns of pathogenic isolates revealed varied resistance to most of the antibiotics employed. Cefotaxime (a new generation cephalosporin antibiotic) was established in this study as the best antimicrobial agent for treatment of PID due to Gram-positive and Gram-negative bacteria isolated from the women examined.

Conclusion: In conclusion, Pelvic inflammatory disease is a major health problem in developed or developing countries of the world. PID is not a notifiable disease, as accurate statistics on disease prevalence are rarely available. There is therefore no doubt thousands of young women have salpingitis every year and their sheer number makes it an important health problem. PID hence can be said to be a very serious complication of sexually transmitted disease which should be critically and promptly handled by healthcare providers. The right type sample should be aseptically collected and be appropriately handled for laboratory investigation. Treatment of PID should be initiated as soon as the presumptive diagnosis has been made. Immediate administration of antibiotics has been effective in the long-term sequelae associated with PID, especially new generation antibiotics, such as cefotaxime as recorded in this study.
\end{abstract}

Key words: Bacterial isolates, Pelvic inflammatory disease, Female patients

\section{Introduction}

Pelvic inflammatory disease refers to any infection in the female lower reproductive tract that spreads to the upper reproductive tract. The female lower genital tract embraces the vagina and cervix while the upper genital tract consists of the uterus, the fallopian or uterine tubes and the ovaries (Landers and Sweet, 1997).

The disease comprises a spectrum of inflammatory disorders of the upper female genital tract, including any combination of endometritis, salpingitis, tubo-ovarian abscess and pelvic peritonitis. Sexually transmitted organisms, especially Neisseria gonorrhoea and Chlamydia trachomatis have been implicated in many cases; however microorganisms that comprise the vagina flora such as anaerobes, Gardnerella vaginalis, Haemophilus influenzae, entetric Gram-negative rods and Streptococccus agalactiae also have been associated with PID. In addition, Cytomegalovirus (CMV), Mycoplasma hominis and Ureaplasma urealyticum may be the etiologic agents in some cases of PID (CDCP, 2002).

If the disease is left untreated, it could result in serious consequences such as infertility, tubal (ectopic) pregnancy, chronic abdominal pain and internal pelvic scarring ( $D^{\prime}$ Hooghe 1999). Pelvic inflammatory disease is generally restricted to women of menstruating age and seldom affects women who do not menstruate ( $D^{\prime}$ Hooghe et al., 1999). According to Ross (2002), in chronic PID, inflammation could extend to other areas of the body and could form pus-filled blisters on the ovaries and fallopian tubes. The blisters if large enough can cause permanent scarring in the reproductive system and thus block the movement of ova from the fallopian tubes to the uterus, thus resulting in infertility or tubal pregnancy. Hill (2002) stated that apart from the basic symptom of lower abdominal or pelvic pain associated with PID which could be constant and intense coupled with sexual intercourse, other symptoms of PID would include, abnormal vaginal discharge, abnormal and or 
heavy vaginal bleeding, bleeding between periods, fever with chills, nausea and vomiting, increased pain during ovulation, lower back pain, fatigues and frequent and painful micturition.

Specific diagnosis of Pelvic inflammatory disease is difficult to make because the upper pelvic organs are difficult to reach for sampling. Samples are taken directly from the cervix to identify the organisms that may be responsible for the infection. Samples can also be taken from the cavity surrounding the ovaries called the cul-de-sac. The fluid so obtained may be examined directly for bacteria or may be used for culture (Landers and Sweet, 1997).

According to Saini et al., (2003), PID is not a notifiable disease in most countries, so accurate statistics are not available. This situation is not in any way different here in Nigeria and more so in the Federal Capital Territory, Abuja where this research was conducted, there had never been any published report so far on PID.

\section{Materials and Methods Microbiological analysis}

One hundred Endocervical specimens were aseptically collected from female patients with certified cases of Pelvic inflammatory disease (PID), attending some hospitals in the Federal Capital Territory, Abuja, Nigeria based on the following criteria:

1. History of recurrent lower abdominal pain.

2. Presence of lower abdominal tenderness.

3. Presence of cervical motion tenderness.

4. Presence of adnexal motion tenderness.

5. Oral temperature $>38^{\circ} \mathrm{C}$ and or leucocytosis $>10,500 / \mathrm{mm}^{3}$.

Direct Examination: Saline wet preparation was carried out in order to rule out the presence of Trichomonas vaginalis which is characteristically associated with a malodorous yellow-green discharge, itching, redness and swelling. Direct Gram's staining was done on smears made from the swab specimens for the characteristic morphological identification of organisms present. Giemsa stain, a Romanowsky stain was employed to stain for the possible presence of Chlamydia trachomatis and Chlamydia granulomatis coccobacilli referred to as Donovan bodies which would stain blue-mauve to dark purple depending on stage of development.

Specimen Cultivation: The Endocervical specimens were respectively cultivated on Blood agar, McConkey agar and Chocolate agar media and incubated aerobically at $37{ }^{\circ} \mathrm{C}$ for $24 \mathrm{~h}$; the Chocolate agar culture was in addition incubated with increased $10 \% \mathrm{CO}_{2}$. Additional Blood agar cultures of specimens were subjected to anaerobic incubation at same temperature and time as the aerobic cultures for possible detection of the presence of obligate anaerobes.

Identification of Isolates: Identification was based on macroscopical examination of growth on cultured plates, microscopical studies of isolates on account of Gram's staining reaction and biochemical characterisation coupled with test for motility.

Antibiogram: The disc diffusion technique was used for the antibiogram while Staphylococcus aureus NCTC 6571 and Escherichia coli NCTC 10418 were used as controls for Gram-positive and Gram-negative bacteria respectively.

\section{Results} (Figure 1).

Out of the 100 samples examined, monomicrobial isolates constituted $43 \%$, polymicrobials, $2 \%$ while sterile cultures constituted $55 \%$

Staphylococcus aureus had the highest occurrence frequency of $14 \%$ followed by Escherichia coli with $9 \%$ while Proteus mirabilis had the lowest occurrence frequency of $1 \%$ (Table 1). In relation to positive cultures, Staphylococcus aureus had the highest percentage occurrence of $34 \%$, followed by Escherichia coli with $21 \%$ percentage occurrence while Proteus mirabilis had the least percentage occurrence of $2 \%$ (Table 2 ). In relation to age, subjects in the age bracket 25-35 years had the highest endocervical positive cultures (30\%) while the least (1\%) was amongst the 36-45 years age group (Table 3). With respect to marital status, the highest rate (90\%) of endocervical infection came from samples from women who belong to the polygamous homes while the least rate (13\%) was observed in the monogamous group (Table 4).

The antibiogram studies on the isolates revealed that Cefotaxime was the most effective compared with all the other antibiotics used against the various endocervical isolates followed by ciprofloxacin (Table 5).

Table 1: Occurrence Frequency of Isolates In Relation To Sample Size

\begin{tabular}{lc}
\hline ORGANISM & FREQUENCY/PERCENTAGE (\%) \\
\hline Staphylococcus aureus & $14(14)$ \\
Streptococcus pyogenes & $3(3)$ \\
Streptococcus faecalis & $7(7)$ \\
Escherichia coli & $9(9)$ \\
Pseudomonas aeruginosa & $4(4)$ \\
Klebsiella pneumoniae & $3(3)$ \\
Proteus rettgeri & $2(2)$ \\
Proteus mirabilis & $1(1)$ \\
Mixed infection & $2(2)$ \\
Sterile culture & $55(55)$ \\
\hline Total & 100
\end{tabular}


Table 2: Percentage Frequency of Pathogenic Isolates In Relation To Positive Cultures $\mathrm{Tn}=47$

\begin{tabular}{lc}
\hline ORGANISM & FREQUENCY/PERCENTAGE (\%) \\
\hline Staphylococcus aureus & $16(34)$ \\
Streptococcus pyogenes & $10(21)$ \\
Streptococcus faecalis & $8(17)$ \\
Escherichia coli & $3(6)$ \\
Pseudomonas aeruginosa & $4(9)$ \\
Klebsiella pneumoniae & $3(6)$ \\
Proteus rettgeri & $2(4)$ \\
Proteus mirabilis & $1(2)$
\end{tabular}

Table 3: Occurrence of Positive Cultures In Relation To Age

\begin{tabular}{lcc}
\hline AGE & $\begin{array}{c}\text { NUMBER } \\
\text { SAMPLED }\end{array}$ & $\begin{array}{l}\text { POSITIVE } \\
\text { CULTURE/ } \\
\text { PERCENTAGE (\%) }\end{array}$ \\
\hline $15-25$ & 27 & $16(16)$ \\
$25-35$ & 68 & $30(30)$ \\
$35-45$ & 5 & $1(1)$ \\
\hline Total & $\mathbf{1 0 0}$ & $\mathbf{4 7}(\mathbf{4 7})$ \\
$\mathbf{X}^{\mathbf{2}}$ Calculated $=\mathbf{1 . 7 6}, \mathbf{X}^{\mathbf{2}}$ Tabulated $=\mathbf{5 . 9 9 1 , ~} \mathbf{d f}=\mathbf{2 , \mathbf { P } > \mathbf { 0 . 0 5 }}$
\end{tabular}

Table 4: Endocervical Isolates in Relation to Marital Status

\begin{tabular}{|c|c|c|c|}
\hline Marital status & Number examined & Bacterial isolates & Frequency/Percentage (\%) \\
\hline \multirow[t]{4}{*}{ Monogamy } & $30 \quad$ Stc & phylococcus aureus & $2(7)$ \\
\hline & Esch & erichia coli & $1(3)$ \\
\hline & Stre & tococcus pyogenes & $1(3)$ \\
\hline & & & 13 \\
\hline \multirow[t]{7}{*}{ Polygamy } & 20 & hylococcus aureus & 7 (35) \\
\hline & Esch & erichia coli & $4(20)$ \\
\hline & Strep & tococcus faecalis & $4(20)$ \\
\hline & Strep & tococcus pyogenes & $1(5)$ \\
\hline & Klebs & iella pneumoniae & $1(5)$ \\
\hline & Pseuc & omonas aeruginosa & $1(5)$ \\
\hline & & & 90 \\
\hline \multirow[t]{9}{*}{ Singles } & Stan & hylococcus aureus & $7(14)$ \\
\hline & Stre & tococcus pyogenes & $1(2)$ \\
\hline & Pseu & Yomonas aeruginosa & $3(6)$ \\
\hline & Strep & tococcus faecalis & $4(8)$ \\
\hline & Prote & us mirabilis & $2(4)$ \\
\hline & Kleb & iella pneumoniae & $1(2)$ \\
\hline & Esch & richia coli & $2(4)$ \\
\hline & Prote & us rettgeri & $5(10)$ \\
\hline & & & 50 \\
\hline
\end{tabular}

Table 5: Susceptibility Frequency Pattern of Bacterial Isolates in the Pelvic Inflammatory Disease Investigated.

\begin{tabular}{lcccccccccc}
\hline ORGANISM & AMP & GEN & NOF & CEFO & CEFTA & ERY & CIPRO & STR & LN & CHLORA \\
\hline Staphylococcus aureus & 6 & 11 & 10 & 14 & 5 & 8 & 14 & 11 & 9 & 1 \\
Streptococcus faecalis & 2 & 1 & 4 & 6 & 7 & 6 & 4 & 4 & 1 & 2 \\
Streptococcus pyogenes & 0 & 1 & 1 & 2 & 1 & 1 & 1 & 1 & 0 & 0 \\
Escherichia coli & 3 & 8 & 9 & 9 & 3 & 2 & 1 & 0 & 1 & 0 \\
Pseudomonas aeruginosa & 0 & 1 & 1 & 1 & 4 & 0 & 1 & 0 & 0 & 0 \\
Klebsiella pneumoniae & 0 & 3 & 3 & 2 & 1 & 0 & 1 & 0 & 1 & 1 \\
Proteus rettgeri & 1 & 2 & 0 & 2 & 1 & 2 & 2 & 0 & 1 & 0 \\
Proteus mirabilis & 0 & 1 & 0 & 1 & 0 & 1 & 1 & 0 & 1 & 0
\end{tabular}

AMP-Ampicillin, GEN-Gentamycin, NOF-Nofloxacin, CEFO-Cefotaxime, CEFTA-Ceftzidime, ERY-Erythromycin, CIPRO-Ciprofloxacin. STRStreptomycin, LN-Lincomycin, CHLORA-Chloramphenicol. 


\section{Discussion}

Pelvic inflammatory disease is a common disease occurring in young women who are sexually active. The bacteriology of acute PID is polymicrobial in nature and this is often associated with a more severe clinical presentation, poor response to therapy, increased risk for more chronic pelvic pain, ectopic pregnancy and infertility. The most common symptoms of patients in this study included lower abdominal pain, abnormal cervical or vaginal mucopurulent discharge with abdominal tenderness, oral temperature $>38^{\circ} \mathrm{C}$, all of which satisfy minimum criteria for diagnosis of PID according to CDCP (1998).

It was observed in this study that the mixed cultures were mainly of Staphylococcus aureus and Streptococcus faecalis isolated in one case and Escherichia coli and Staphylococcus aureus in another with $2 \%$ occurrence respectively, whereas the monomicrobials presented with $43 \%$ occurrence. This finding differs from previous works as recorded by Saini et al. (2003) in India on 50 women with history and examination suggestive of PID of polymicrobial origins shown to constitute $43.2 \%$ and monomicrobial origins, $56.7 \%$. This difference in findings is not clearly understood, though could be attributed to variance in geographical location or the high population density in India which probably could engender promiscuity with the possible attendant effect of acquisition of PID. However, the findings agree in part with those of same authors who isolated Staphylococcus aureus and Escherichia coli as the predominant aerobic isolates with $57.4 \%$ occurrence and this correlates with previous studies of Ayyagari et al. (1987) and Gupta (1978) who had 54\% occurrence of the organisms respectively. The slight difference in isolation rates could probably be attributed to feminine diversity in social behaviours and exposure. Also the difference could be attributed to sample type variation in which the Pouch of Douglas (cul-de-sac) aspirate which presents with a better diagnostic precision were used by these authors as opposed to endocervical swab procedure used in this study.

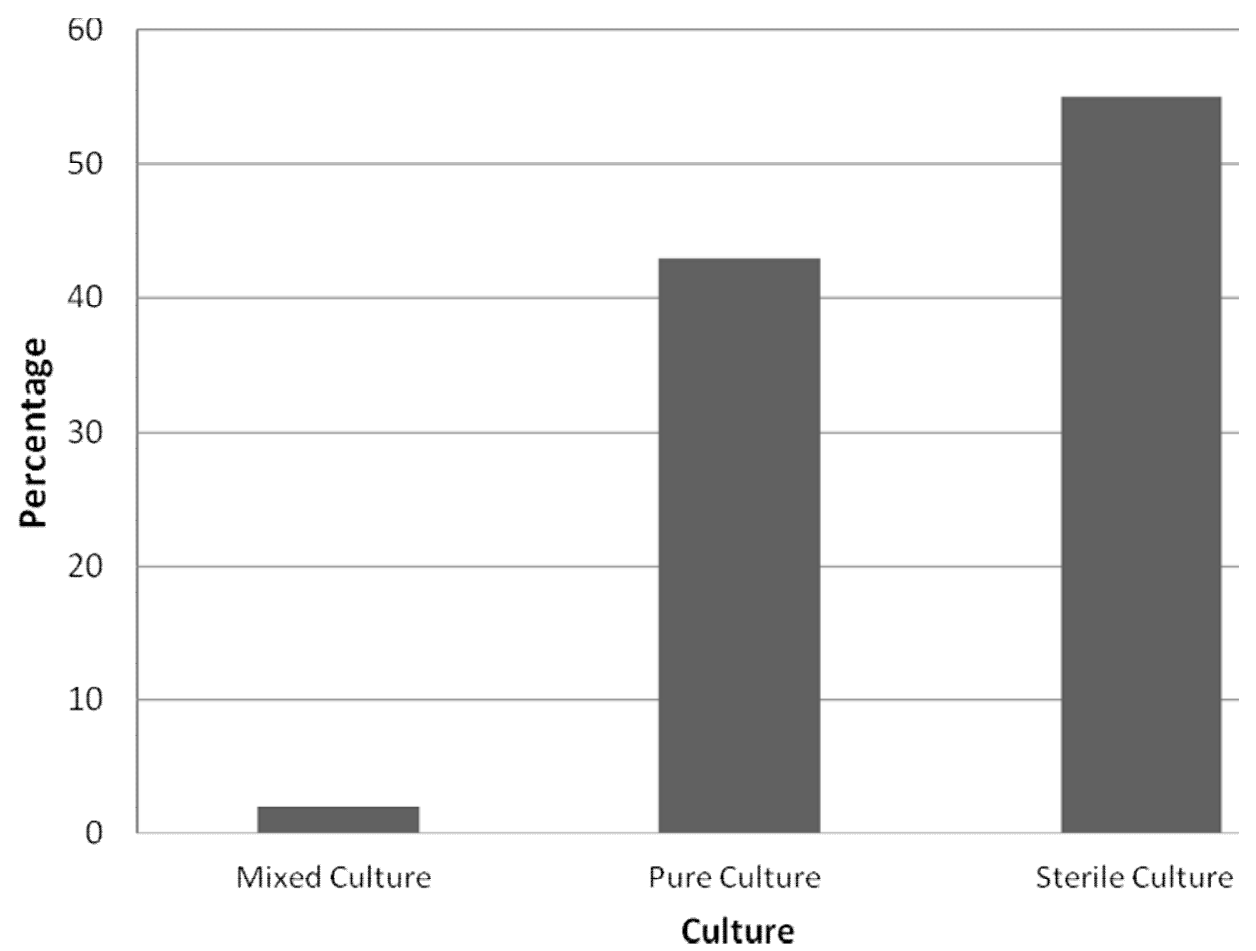

KEY

Figure 1: Histogram of frequency of occurrence of Mixed, Pure and Sterile cultures

Mixed Culture - 2\%, Pure Culture - 43\%, Sterile Culture - 55\%

Endocervical isolates were recovered much more in the polygamous patients (90\%) than in the unmarried or monogamous patients. This is in agreement with the finding of NIH (1997), that PID occurs less readily in women with monogamous relationships than in the unmarried or polygamous women. There was a significant difference $(P<0.05)$ in the occurrence of PID with respect to marital status which goes to buttress the fact as revealed in this study that polygamy is one of the major risk factors in the acquisition of PID.

The age range of 26-35 years accounted for the highest occurrence of PID in this study with 68 cases as compared to age groups 15-25 years (group that is supposed to be most vulnerable to PID because of their high sexual activity) and 36-45 years with 27 and 5 cases respectively. The reason for the very high occurrence of PID in the age range of 26-35 years is not clearly understood. However, it is believed that majority of subjects in this age bracket are singles, either in the universities or other tertiary institutions of learning. It is possible they practise sexual relationships involving multiple sexual partners with resultant high rates of abortion as sequelae to promiscuity. Only few of them might probably have reported to hospitals for treatment or counselling due to poor or complete lack of awareness on the need for regular medical check-ups .

Subjects within the age range 36-45 years are women who are either married with children and/or with good jobs, singles or divorcees with good socio-economic status and hence capable of taking good care of themselves medically; thus are less frequently affected as revealed in this study. However, there was no significant difference $(P>0.05)$ in the occurrence of PID with respect to age which goes to show that any of the age groups investigated could be vulnerable to the disease. 
The most common primary infecting organisms such as Chlamydia trachomatis and Neisseria gonorrhoea, as recorded by CDCP (2002), were not encountered in this study, as the organisms isolated were mainly aerobic bacteria and facultative anaerobes. This difference in microbial variation could not be clearly understood. However, it could be seen that apart from already established organisms in PID cases, aerobic bacteria or facultative anaerobes which might have supplanted the vaginal microflora (the lactobacilli) could also be implicated as agents of PID as encountered in this study.

The antibiogram study showed increased antimicrobial resistance by both the Gram-negative and Gram-positive bacteria respectively as revealed in this study to the wide array of antimicrobials employed. In general, cefotaxime, ciprofloxacin and streptomycin had mean cidal effects of $77 \%, 57 \%$ and $51 \%$ respectively against all the Gram-positive bacteria encountered while gentamycin, cefotaxime and ciprofloxacin had mean cidal effects of $80 \%, 76 \%$ and $54 \%$ respectively against all the Gram-negative bacteria encountered. These results indicate that cefotaxime can be used as a general frontline antibiotic to treat cases of PID involving both Gram-positive and Gram-negative bacteria respectively. This assertion correlates with CDCSP (2002). Treatment Guidelines on the treatment of STDs with respect especially to PID with cefotaxime, a new third generation cephalosporin.

The increased resistance to antibiotics as encountered in this study could be explained by indiscriminate antimicrobial prescription practices, nosocomial transmission of resistant organisms and changes in social conditions such as urban crowding. Obligate anaerobic bacteria and few other organisms associated with PID other than the ones isolated were not encountered in this study. This therefore, calls for sophisticated testing procedures such as sonogram, endometrial biopsy, laparoscopy etc. by healthcare providers, especially in developing countries.

Prevention: Pelvic inflammatory disease (PID) may be prevented thus:

- Abstinence from sexual intercourse

- Safe sex practice which includes the use of condoms and avoidance of multiple sexual partners.

- Get tested if at risk of a Sexually transmitted infection e.g. chlamydiasis or gonorrhoeae.

- Getting early treatment which may prevent development of PID.

- Avoidance of douching as this upsets the delicate balance of vagina microflora.

- Pay attention to good hygiene habits - wipe from front to back after urinating or having bowel movement to avoid introducing bacteria from colon to vagina.

In conclusion, PID is a major health problem in developed or developing countries of the world. Despite advances in defining its aetiology, pathogenesis and availability of many potent antimicrobial drugs, it consumes a significant proportion of medical resources of numerous countries. In many countries, PID is not a notifiable disease, as accurate statistics on disease prevalence are rarely available. There is, therefore, no doubt thousands of young women have salpingitis every year and their sheer number makes it an important health problem. PID hence can be said to be a very serious complication of sexually transmitted disease which should be critically and promptly handled by healthcare providers. Pelvic examination in cases of PID should be carried out by the physician and if this reveals tenderness or pain in that region or tenderness on movement of the cervix, it is suggestive of PID. The right type sample should be aseptically collected and be appropriately handled for laboratory investigation. Treatment of PID should be initiated as soon as the presumptive diagnosis has been made. Immediate administration of antibiotics has been effective in the long-term sequelae associated with PID.

\section{References}

1. Ayyagari, A; Chakrabarti, A; Singh, A; Sapru, A; Aggarwal, K. C. (1987). Bacteriology of diverse infections of female genital tract with particular reference to anaerobic bacteria. Indian Journal of Medical microbiology, 5: 189-195.

2. Center for Disease Control and Prevention (1998). Guidelines for control of sexually transmitted diseases. Morbidity and Mortality Weekly Report, 51, 47(RR-1): 1-11.

3. Center for Disease Control and Prevention (2002). Pelvic inflammatory disease. Sexually transmitted disease treatment guidelines. Morbidity and Mortality Weekly Report, 51(RR-6): 48-52.

4. D' Hooghe, T. M; Bambra, C. S; Raeymacker, B. M; Hill, J. A. (1999). Pelvic inflammation induced by Diagnostic laparoscopy in baboons. Fertility and Sterility, 72(6): 1134-1140.

5. Gupta, V. (1978). Anaerobic bacteria in human infection - A review. Indian Practitioner, 31: 271-289.

6. Hills, A.D. (2002). Pelvic inflammatory disease. Issues and procedures in woman health, Pp 1-3.

7. Landers, D. V. and Sweet, R. L. (1997). Pelvic inflammatory disease. Eds. New York: Springer.

8. National Institute of Health (1997). Pelvic inflammatory disease. Fact Sheet Guidelines for Treatment, Prevention and Management.

9. Ross, J. (2002). Pelvic inflammatory disease. Clinical Evidence, 7:1452-1457.

10. Saini, S. N. N Gupta, Aparna, G Batra, DR Arora (2003). Role of anaerobes in acute Pelvic inflammatory disease. Indian Journal of Medical microbiology, 21(3): 189-192. 